\title{
The effects of instructions on scales for perceived egocentric distance in a large open field
}

\author{
JOSÉ APARECIDO DA SILVA and RAQUEL ALVES DOS SANTOS \\ University of São Paulo, Ribeirão Preto, Brazil
}

\begin{abstract}
Data from a magnitude estimation study of distance in a large open field are presented. Two separate sets of instructions intended to induce either physical or apparent attitudes for reporting the magnitude estimates of distance were employed in two experiments. The results showed that the type of instructions, regardless of the degree of availability of a standard distance in the series of judgments, does not affect the exponent of the power function relating perceived distance to physical distance. The mean exponent for all 80 individual power functions was 0.87 , with a standard deviation of 0.09 .
\end{abstract}

The scale for perceived distance frequently has been described by the power function $\mathrm{D}^{\prime}=\mathrm{KD}^{\mathrm{n}}$, where $\mathrm{D}^{\prime}$ is the perceived distance, $D$ is the physical distance, $K$ is a constant defining the scale unit, and $\mathrm{n}$ is the exponent. This exponent is an important parameter that reflects the acceleration of the function relating distance estimates to physical distance and indicates the degree of distance constancy (Baird, 1970; Da Silva \& Dos Santos, 1982). Perceived distance is a linear function of physical distance when $\mathrm{n}$ equals one, and in that case perfect constancy is said to occur. On the other hand, if $n>1.0$, there is a positive acceleration in the function that indicates overconstancy, and if $n<1.0$, there is a negative acceleration that indicates underconstancy (Baird, 1970; Da Silva, 1983a; M. Teghtsoonian \& Beckwith, 1976).

Most previous studies have employed almost exclusively the apparent-distance instructions to scale perceived distance. In fact, Carlson (1977) noted that the effects of instructions on perceived distance have not been investigated systematically. Using apparent-distance instructions, R. Teghtsoonian and M. Teghtsoonian (1970a) found a function positively accelerated, which indicated overconstancy. Their observers made magnitude estimates of distance that varied from 5 to $45 \mathrm{ft}$ in a large room. Overconstancy was. also found by $M$. Teghtsoonian and R. Teghtsoonian (1969) in two other experiments, one performed in a large room and the

This research was supported by Grants 10.469-79 and 10.47079 from the National Research Council of Brazil (CNPq) and was prepared for publication while the first author was a visitor at the Department of Psychology, University of California, Santa Barbara. The preparation of the manuscript was supported by Grant 82/0033-6 from the Fundação de Amparo à Pesquisa do Estado de São Paulo (FAPESP). Thanks are due Walter C. Gogel and Jack M. Loomis for useful discussions and suggestions on an earlier version of this article. The authors' mailing address is: Department of Psychology, University of São Paulo, 14.100 Ribeirão Preto, São Paulo, Brazil. other in a corridor. They used distances that varied from 5 to $80 \mathrm{ft}$ and used apparent-distance instructions. In another experiment carried out in a natural outdoor setting, R. Teghtsoonian and M. Teghtsoonian (1970b) employed ranges that varied from 37 to $480 \mathrm{ft}$. They found perfect constancy with the $37-\mathrm{ft}$ range and underconstancy with two larger ranges. A positively accelerated function was also found by $\mathbf{R}$. Teghtsoonian and M. Teghtsoonian (1978) in experiments that were carried out in a large room and that specified apparent distance in the instructions.

Da Silva (1983b) and M. Teghtsoonian and Beckwith (1976), discussing these and other experiments, pointed out that the environment in which the distances are judged probably affects somewhat the function relating perceived distance to physical distance. Perceived distance is an accelerating function of physical distance in a natural indoor setting, and it is a decelerating function in a natural outdoor setting. On the other hand, it may be as Carlson (1977, p. 241) suggested, that the instructions had been the principal variable responsible for the kind of function relating judged distance to physical distance in those experiments. Carlson noted that there was a change in instructions in the Teghtsoonian and Teghtsoonian experiments. In fact, $\mathbf{M}$. Teghtsoonian and R. Teghtsoonian (1969) used apparent-distance instructions in experiments performed in an indoor setting and neutral-apparent-distance instructions in experiments performed in an outdoor setting (R. Teghtsoonian \& M. Teghtsoonian, 1970b). According to Carlson's analysis, this subtle difference in instructions can often account for larger differences in results, and it may be as important for distance estimates as it is for size judgments. The two experiments described below were designed to test this hypothesis, since the effects of instructions on perceived distance had not been studied with the magnitude estimation procedure.

Only in the study by Rogers and Gogel (1975) were 
the effects of instructions on perceived distance investigated. In that study, apparent-distance and objectivedistance instructions were used with several tasks involving the judgment of distance, including verbal report. The distances varied from 0.69 to about $7 \mathrm{~m}$. Their results showed that, in a bisection task, the mean exponent of the power function was significantly larger for objectivethan for apparent-distance instructions. Apparentdistance instructions produced approximately perfect distance constancy, whereas objective-distance instructions resulted in overconstancy. However, the difference between the two mean exponents was not very large. For verbal-report and interval-reproduction tasks, judgments of distance did not differ significantly as a function of instructions. Furthermore, overconstancy was produced only by the combination of objective-distance instructions and the bisection task, and under-constancy was produced only by the combination of apparentdistance instructions and the verbal-report method (Rogers \& Gogel, 1975, p. 177).

Gogel (1974) suggested that such a tendency to overconstancy is the result of inferential or cognitive factors. He suggested that this overconstancy reflects an inappropriate application of a cognitive correction by an observer in an effort to be correct in distance judgments. However, reponse errors can occur despite the cognitive correction, depending on both the manner in which the corrections are applied to perceived distance and the manner in which perceived distance varies with physical distance. In support of this interpretation, Gogel (1974) presented evidence indicating that, at relatively near distances, an observer usually applies a large correction that results in reports of overconstancy and, at far distances, applies a small correction that results in reports of underconstancy. Also, consistent with this analysis, it might be expected that the amount of correction and thus the amount of overconstancy would be less when an observer is instructed to respond to apparent distance rather than to physical distance. In other words, this tendency to overconstancy can be reduced by using instructions that minimize the need for an observer to make physically accurate distance judgments (Gogel, 1974; Rogers \& Gogel, 1975).

The present investigation was undertaken to determine whether the function relating magnitude estimates of distance to physical distance is affected by the type of instructions given to observers. The two experiments reported in this paper represent an extension of the studies of Da Silva and Da Silva (1983) and Da Silva and Dos Santos (1982). These two experiments contrast the results obtained with two sets of instructions inducing apparent and objective attitudes to estimate distances in a large open field. In Experiment 1, magnitude estimates of distance were made with a standard distance presented only at the beginning of each series of judgments. In Experiment 2, magnitude estimates were performed with a standard distance shown throughout the experiment.

\section{EXPERIMENT 1}

\section{Method}

Observers. The observers were 40 students, between 17 and 29 years old, who partially satisfied a course requirement in introductory psychology by participating in the experiment. All had a visual acuity of at least 20/20, near and far (corrected if necessary), in each eye, as measured on an orthorater. They were naive with regard to the nature of the experiment and were not familiar with the experimental field.

Materials and Viewing situation. The viewing situation was a large open field $300 \mathrm{~m}$ long and $30 \mathrm{~m}$ wide and especially landscaped to be appropriate for this kind of experiment. There were small trees and bushes at the sides and at the far end. At the central line of the field, seven distances were inconspicuously marked, so the experimenter could identify them, at $1.60,3.20$, $6.40,12.80,25.60,51.20$, and $102.40 \mathrm{~m}$, encompassing a range of $1.80 \mathrm{log}$ units as defined by R. Teghtsoonian (1973). The distances were marked by seven different-sized yellow triangles, ranging in size from $14.70 \mathrm{~cm}$ (base) $\times 13 \mathrm{~cm}$ (height) to $105 \mathrm{x}$ $120 \mathrm{~cm}$. Only one triangle was placed at a time, and it was in the frontal-parallel plane. All distances were marked from the observer. An assistant placed the triangles for the three farthest distances, another assistant placed the triangles for the four nearest distances, and the experimenter, who was beside the observer, recorded the magnitude estimates given by the observer and closed the observation window while the distances were changed.

Procedure. The observer remained seated on a piano bench (adjustable in height), with his or her head fixed in a chinrest. The average height of an observer's eyes from the ground was $110 \mathrm{~cm}$. The observers could survey the field and its surroundings binocularly through a large centered window $(29 \times 26 \mathrm{~cm})$ around the station point used in static viewing. This window was closed manually by the experimenter while changes of distances were made.

The 40 observers in this experiment were divided into two groups of 20 each. One group made magnitude estimates under instructions to estimate the apparent distance, and the other group received instructions to judge the objective distances of the different triangles presented one at a time. The instructions were adapted, with few modifications, from those used by M. Teghtsoonian (1965, pp. 393-394) to scale area, volume, and length. They were intended to induce either apparent or physical attitudes for reporting the magnitude estimates of distance.

For apparent-distance judgments, the observers received the following instructions, individually given:

I will show you a series of distances which will be marked by different sized triangles. Your task will consist in telling how far these triangles appear to be from your eyes, by assigning numbers to them. First, I will show you a triangle whose apparent distance from your eyes will be arbitrarily called 50. Then, when I show you the other distances one at a time, I want you to assign a number to each one proportional to its apparent distance. For example, a distance which appears to be twice as large as that of the one called 50 would be called 100. A distance which appears to be half as large would be called 25 , and so on. You must always compare each distance with the standard distance. Don't try to be consistent, but assign a number which represents how far the triangle appears to be from your eyes. Please disregard any information you may have about the physical distance of the triangles. I am only interested in your reporting how far the triangle appears to be from your eyes and not where you think or guess it is physically. You may use any number that seems appropriate-whole number, fractions or decimal. Don't try to remember what number you assigned to preceding distances. Do you have any questions? Then, we'll start. [Standard distance presented.] This is the triangle 
whose apparent distance from your eyes is called 50. [Standard removed, and first distance presented.] If the first apparent distance was 50 , what would you call this one?

The observers who were to respond to objective characteristics received the following instructions:

I will show you a series of distances which will be marked by different sized triangles. Your task will consist in telling how far you judge they are physically from your eyes, by assigning numbers to them. First, I will show you a triangle whose physical distance from your eyes will arbitrarily be called 50. Then, when I show you the other distances one at a time, I want you to assign a number to each one which is proportional to its physical distance. For example, a distance which is physically twice as large as that of the one called 50 would be called 100 . A distance which is physically half as large would be called 25 , and so on. You must always compare each distance with the standard distance. Don't try to be consistent, but assign a number which represents how far the triangle is physically located from your eyes. Please try to assign a number proportional to your objectively estimated impression. I am only interested in your reporting how far you think the triangle is physically from your eyes, not how far it appears to be from your eyes. You may use any number that seems appropriate-whole number, fractions or decimal. Don't try to remember what number you assigned to preceding distances. Do you have any questions? Then, we'll start. [Standard distance presented.] This is the triangle whose physical distance from your eyes is called 50. [Standard removed, and first distance presented.] If the first physical distance was 50 , what would you call this one?

The method of magnitude estimation with a modular distance of $6.40 \mathrm{~m}$ was used. This modular distance (standard distance) was shown only at the beginning of each series of judgments and was identified with the modulus 50. A series of judgments consisted in magnitude estimates of the 6 distances, with $6.40 \mathrm{~m}$ being used as the standard distance. The standard was present for $10 \mathrm{sec}$. The other distances (excluding the standard distance) were presented one at a time, in a different order for each observer. Each distance was presented until the observer had made a judgment. Each distance was judged twice.

\section{Results}

For each observer, a straight line was fitted by the method of least squares, relating $\log$ magnitude estimates to $\log$ actual distances. The assigned modulus was treated as a judgment (equal to 50). Its slope, which is the exponent of a power function, and the scale factor were determined for each group. The arithmetic means and standard deviations of the exponents for each type of instruction were: $0.88 \pm 0.08$ (range $=0.75$ to 1.02 ) for objective-distance instructions, and $0.85 \pm 0.08$ (range $=0.71$ to 1.01 ) for apparent-distance instructions. The scale factors were: $0.18 \pm 0.08$ (range $=0.07$ to 0.36 ) for objective-distance instructions, and $0.23 \pm 0.11$ (range $=0.08$ to 0.51 ) for apparent-distance instructions. The good quality of the fits for individual observers is indicated by the mean coefficients of determination, $\mathrm{r}^{2}$, for individual fitted lines: $r^{2}=0.99$ for both types of instructions.

A t test revealed that the mean exponent for objectivedistance instructions was not different from that for apparent-distance instructions $[\mathrm{t}(38)=1.54, \mathrm{p}>.10]$. A similar test indicated that the mean scale factor for objective-distance instructions was not different from that for apparent-distance instructions $[\mathrm{t}(38)=1.69$, $\mathrm{p}>.05$ ]. In addition, $t$ tests showed that both mean exponents were less than a theoretical value of 1.0 (ps <.001). Combining the two groups of observers, the exponent was smaller than 1.0 for approximately $92 \%$ of the pooled sample.

\section{EXPERIMENT 2}

Da Silva and Da Silva (1983) and Da Silva and Dos Santos (1982) showed that the availability of a standard throughout the experiment, regardless of the distance range, does not affect the exponent of the power function that relates the magnitude estimates of distance to physical distance for large distances in an open field. However, in both studies, only apparent-distance instructions were employed. Experiment 2 was designed to determine how the presence of the standard distance throughout the experiment interacts with the type of instructions. As in Experiment 1, apparent-distance and objective-distance instructions were again employed, but the standard distance was presented throughout the experiment.

\section{Method}

Observers. Forty students between 17 and 27 years old participated in this experiment. All participants had normal or corrected-to-normal vision. The participants had had no previous exposure to experiments in visual perception and were not aware of the purpose of the study.

Viewing situation and Procedure. The experimental situation and the materials employed were essentially the same as those used in Experiment 1. As in Experiment 1, the same seven different-sized yellow triangles, ranging in size from $14.70 \mathrm{~cm}$ at the base $\mathrm{x} 13 \mathrm{~cm}$ in height to $105 \mathrm{~cm} \mathrm{x} 120 \mathrm{~cm}$, were used to indicate the distances. Also, the procedure was identical to that of Experiment 1, except that: (1) The following distances were used-2, 4, 8, 16, 32, 64, and $128 \mathrm{~m}$, encompassing an equal range of $1.80 \mathrm{log}$ units. This means that the absolute distances were different from those of Experiment 1, but the ratio was constant. (2) The standard distance $(8 \mathrm{~m}$, identified with the modulus 50) was presented throughout the experiment.

The 40 observers in this experiment were divided into two groups of 20 each. One group received apparent-distance instructions and the other group received objective-distance instructions to make magnitude estimates of the comparison distance relative to the standard distance during the presentation of the comparison distance. The instructions were identical to those employed in Experiment 1.

\section{Results}

The data from this experiment were analyzed in the same way as those from Experiment 1 . The exponents of the individual observers were estimated by the slopes of the best-fitting straight line relating log magnitude estimates to log physical distances. The mean exponents and standard deviations for the two groups of observers were: $0.88 \pm 0.11$ (range $=0.59$ to 1.04 ) for objectivedistance instructions, and $0.87 \pm 0.07$ (range $=0.73$ to 1.01) for apparent-distance instructions. The mean scale factors were: $0.16 \pm 0.08$ (range $=0.06$ to 0.37 ) and $0.19 \pm 0.19$ (range $=0.05$ to 0.37 ) for apparent-distance and objective-distance instructions, respectively. The coefficients of determination, $\mathrm{r}^{2}$, indicating the good- 
ness of fit of the two functions, were 0.99 for objectiveand 0.98 for apparent-distance instructions.

A $t$ test indicated that the mean exponent for the objective-distance instructions was not different from that for the apparent-distance instructions $[t(38)=0.21$, $\mathrm{p}>.10]$. A t test also indicated that the mean scale factor for objective-distance instructions was not different from that for apparent-distance instructions $[t(38)=$ $0.72, p>.10]$. A t test showed also that the mean exponents for both types of instructions were less than a theoretical value of 1.0 (ps $<.001$ ). Combining the two groups of observers, the exponent was smaller than 1.0 for approximately $90 \%$ of the pooled sample.

Since the same range of distance in log units was used in both experiments, the individual exponents were pooled and analyzed by means of a two-way analysis of variance (presence/absence of the standard $x$ instructions). This analysis indicated that the effect of the instructions was nonsignificant $[F(1,76)=1.29, p>.05]$, as were the effect of the presence of the standard $[F(1,76)=0.18, p>.05]$ and the interaction between the presence of the standard and the type of instructions $[F(1,76)=0.64, p>.05]$. Thus, the individual exponents from the two experiments can be pooled. The mean exponent was 0.87 , with a standard deviation of 0.09 .

\section{DISCUSSION}

We derived two main findings from the present two experiments: (1) The type of instructions does not affect the mean exponent of the power function that relates magnitude estimates of distance to physical distances. This means that, with the magnitude estimation procedure, apparent- or objective-distance instructions do not produce different distance judgments as a function of physical distance. These results are congruent with those obtained by Gogel and Da Silva (1983), who used the verbal-report procedure. In the Gogel and Da Silva study, distances varying from 40 to $270 \mathrm{~cm}$ were estimated in a multiplecue alley under apparent-distance and objective-distance instructions. Their results showed that the mean exponent for objectivedistance instructions $(n=1.02)$ was not different from that for apparent-distance instructions $(n=1.00)$. Also, the mean scale factor did not differ as a function of instructions. The results of the two experiments described here contradict in part those of Rogers and Gogel (1975), who showed that, with the bisection procedure, apparent-distance instructions tend to produce lower values of $\mathrm{n}$ than do objective-distance instructions. With the verbal-report and interval-reproduction procedures, Rogers and Gogel did not find a significant effect of instructions on distance judgments. On the other hand, our results do not support Carlson's (1977) hypothesis that the change in instructions in the Teghtsoonian and Teghtsoonian experiments was responsible for the different exponents obtained. (2) The lack of an effect of instructions holds both for magnitude estimates made when the standard distance was shown only at the beginning of each series of judgments and for magnitude estimates made when the standard distance was presented throughout the experiment.

Taken together with those of Da Silva and Da Silva (1983) and Da Silva and Dos Santos (1982), the present results confirm the finding that the presence of the standard throughout the experiment, regardless of the type of instructions and of the dis- tance ranges, does not affect the exponent of the power function for perceived distance in the full-cue condition. They also confirm those of Da Silva and Da Silva (1983), Da Silva and Dos Santos (1982), and R. Teghtsoonian and M. Teghtsoonian $(1970 \mathrm{~b})$, who found a mean exponent smaller than 1.0 for the power function relating perceived distance to physical distance for outdoor viewing. As we emphasized, an exponent of less than 1.0 was expected, because, according to an analysis by Gogel (1974), the effectiveness of the major distance cues decreases with increasing physical distance. From this point of view, in a situation involving large distances, perceived distance probably is a decelerating function of physical distance.

\section{REFERENCES}

BAIRd, J. C. (1970). Psychophysical analysis of visual space. New York: Pergamon.

Carlson, V. R. (1977). Instructions and perceptual constancy judgments. In W. Epstein (Ed.), Stability and constancy in visual perception: Mechanisms and processes (pp. 217-254). New York: Wiley.

DA Silva, J. A. (1983a). Scales for measuring subjective distance in children and adults in a large open field. Journal of Psychology, 113, 221-230.

DA Silva, J. A. (1983b). Scales for perceived egocentric distance in a large open field: Comparison of three psychophysical methods. Manuscript submitted for publication.

DA Silva, J. A., \& DA Silva, C. B. (1983). Scaling apparent distance in a large open field: Some new data. Perceptual and Motor Skills, 56, 135-138.

Da Silva, J. A., \& Dos Santos, R. A. (1982). Scaling apparent distance in a large open field: Presence of a standard does not increase the exponent of the power function. Perceptual and Motor Skills, 55, 267-274.

Gogel, W. C. (1974). Cognitive factors in spatial responses. Psychologia, 17, 213-225.

Gogel, W. C., \& DA Silva, J. A. (1983). Effect of instructions on the familiar size cue of distance. Manuscript submitted for publication.

Rogers, S. F., \& Gogel, W. C. (1975). The relation between a judged and physical distance in multicue conditions as a function of instructions and tasks. Perceptual and Motor Skills, 41, 171-178.

Teghtsoonian, M. (1965). The judgment of size. American Journal of Psychology, 78, 392-402.

Teghtsoonian, M., \& Beckwith, J. B. (1976). Children's size judgments when size and distance vary: Is there a developmental trend to overconstancy? Journal of Experimental Child Psychology, 22, 23-39.

Teghtsoonian, M., \& Teghtsoonian, R. (1969). Scaling apparent distance in natural indoor setting. Psychonomic Science, 16, 281-283.

Teghtsoonian, R. (1973). Range effects in psychophysical scaling and a revision of Stevens' law. American Journal of Psychology, 86, 3-27.

Teghtsoonian, R., \& Teghtsoonian, M. (1970a). The effects of size and distance on magnitude estimation of apparent size. American Journal of Psychology, 83, 601-612.

Teghtsoonian, R., \& Teghtsoonian, M. (1970b). Scaling apparent distance in natural outdoor setting. Psychonomic Science, 21, 215-216.

Teghtsoonian, R., \& Teghtsoonian, M. (1978). Range and regression effects in magnitude scaling. Perception \& Psychophysics, 24, 305-314.

(Revision received for publication March 7, 1984.) 